inwards. When the peritoneum was opened thickened cedematous omentum presented itself and the incision was enlarged one and a half inches. In the mid-line was a mass of omentum, underneath which was a gangrenous appendix and a small quantity of very offensive pus. The appendix was removed, the abscess cavity was swabbed out, and a portion of thickened omentum was cut away. The cavity was dusted with iodoform powder and was packed with iodoform gauze, and the greater part of the incision was brought together with silkworm-gut sutures. The iodoform gauze was removed three days after operation and a small piece was reinserted into the cavity for drainage purposes. The cavity filled rapidly and the patient was discharged on August 12th. The patient was again operated upon on Jan. 25th, 1901, for small ventral hernia.

CASE 2. - The patient, a girl. aged 13 years, was admitted into the Metropolitan Hospital on Oct. 11th, 1900. Operation was performed on the 16th and she was discharged on Nov. 13th. The patient had been taken suddenly with abdominal pain on Oct. 2nd. She kept about until the 7 th when she went to bed and diarrhoea commenced. She was admitted into the hospital as suffering from enteric fever. When she was seen after admission a definite swelling in the right iliac fossa was discovered and appendicitis was diagnosed. At the operation an abscess was found situated between the adherent small intestines and creum. This was treated in the manner described above. An uninterrupted recovery followed. Peroxide of hydrogen was used as a lotion.

CASE 3.- The patient, a girl. aged 15 years, was admitted into Sutton Cottage Hospital on Sept. 4th, 1900. Operation was performed on the 9th and the patient was discharged on Oct. 9th. She had been taken ill with abdominal pains and vomiting on Sept. 2nd; the pain and resistance were most marked in the right iliac fossa. When she was seen on the 9th there was a well-marked swelling in the right iliac fossa, the temperature being $103.2 \% \mathrm{~F}$.. but the patient waslying on the affected side reading a book. At the operation an abscess was found containing nearly one ounce of characteristic pus. This was treated on the same lines as the others. The patient made an uninterrupted recovery.

CASE 4. - The patient, a youth. aged 17 years, was admitted into the Metropolitan Hospital on Feb. 6th, 1900. Operation was performed on the $23 \mathrm{rd}$ and the patient was discharged on April 3rd. He had been seized with acute pain in the lower abdomen during ing. The pain and daily vomiting continued until his admission into the hospital, when an indefinite swelling was found in the right iliac fossa extending as far as the middle line. This swelling was dull on percussion. At the operation the omentum. which presented through the wound, was found adherent to the anterior abdominal wound and also to a loop of small intestine; this loop was adherent to the lower part of the cæcum. Between these coils was a small abscess and the remains of the appendix. These were removed and the cavity was packed with gauze. The patient made a good recovery, but the sinus was longer than usual in filling up. Peroxide of oxygen was used as a lotion.

CASE 5. - The patient, a youth, aged 17 years, was admitted into Charing Cross Hospital on Oct. 26th, 1900. An operation was performed on the 26 th and he was discharged on Jan. 9 th, 1901. The previous history of this patient was not clearly made out, but on admission he was quite ill and had obvious signs of suppurating appendicitis. At the operation the omentum, the small intestines, and the cxcum were all matted together, and on separating these a considerable abscess cavity was exposed filled with foul pus. In the cavity was the gangrenous appendix; this was removed and the cavity was swabbed out and packed with iodoform gauze. A portion of the sodden and thickened omentum was removed. The patient did very well until the third week, when he had symptoms of intestinal obstruction, necessitating a laparotomy, which was performed in the mid-line, when two coils of intestine were found adherent to the great omentum. These were separated and the patient made a good recovery.

CAsE 6. - The patient, a girl, aged 11 years, was admitted into the Metropolitan Hospital on July 1st, 1901. Operation was performed on the 2nd and she was discharged on August 4th. She had first complained of pain in the right iliac fossa on June 25th, which gradually became worse until the 29th. There had been no sickness. The bowels had been opened twice a day. On the day of her admission into the hospital she had lamb and mint-sauce twice. At this time her temperature was $103^{\circ} \mathrm{F}$. and her pulse was 132 . The abdomen had a distinct fulness and sense of resistance in the right iliac and lumbar regions, where manipulation caused pain. On opening the abdomen the colon presented itself. This was pulled towards the middle line and an abscess cavity situated externally to the colon was found, which held two ounces of foul pus. The cavity extended up towards the liver and was lined with a thick white membrane. A posterior incision for better drainage was made in the loin and the abscess cavity was treated in the same way as the other cases. Recovery was uninterrupted.

CASE 7.-The patient, a female, aged 48 years, was admitted into the Metropolitan Hospital on July 3rd, 1900. Operation was performed on the 4 th and the patient was discharged on August 14th. She had suffered from sudden severe pain in the lower abdomen on June 25th which settled in the right side; there was no sickness, but rather violent retching. The abdomen moved freely on respiration and on palpation a large mass could be felt in the right iliac and lumbar resions which extended upwards to the lower surface of the liver. The abdomen was opened by an incision four and a half inches in length. When the transversalis muscle was reached it was found to be much infiltrated and thickened. On opening the abscess cavity firm adhesions formed definite and safe boundaries (the only case of this series where this was so). The abscess cavity was treated in the usual manner. Recovery was uninterrupted.

CAsE 8. - The patient. a young man. aged 20 years, was admitted into the Metropolitan Hospital on Nov. 5th, 1901. Operation was performed on the same day and the patient was discharged on Jan. 4th, 1902. He had suffered from a sudden attack of pain with shivering and nausea on Nov. 3rd, 1901, since when the bowels had not acted and no flatus had been passed. Vomiting commenced on the 5th. On admission the patient looked very ill. The temperature was $97.5^{\circ} \mathrm{F}$. and the pulse was 120 . The abdomen was distended and rigid; it did not move with respiration and was very tender all over the right iliac fossa. The feet and hands were found to be peeling in a manner suggestive of scarlet fever. When the patient was sent into the hospital the case was notified as one of typhoid fever. On the abdomen being opened the intestines in the right iliac fossa were found to be covered with a thin puriform fluid. There were no signs of adhesion or appearance of localisation of peritonitis. A long, swollen appendix was found lying on the outer side of the cæcum, between it and the parietal peritoneum. This was removed and a quantity of sero-purulent fiuid was withdrawn by a syringe from the right side of the pelvis. The wound was treated as in the other cases, with the addition that a large drainagetube was passed down into the recto-vesical pouch. The patient was constantly sick until Nov. 8th when, after repeated treatment, the bowels acted well. Recovery after this was uninterrupted. When on the 15th the stitches were removed it was found that no union had taken place, the whole wound gaping and exposing the intestines. No ill effects followed this unusual contretemps.

Harley-street, w.

\section{INTUSSUSCEPTION OF A DIVERTICULUM WITH SECONDARY ILEO-COLIC INTUSSUSCEPTION.}

BY FRED. T, TRAVERS, B.S. LOND., F.R.C.S. EDIN., SLRGFON TO THE WEST KFYT GEXERAL HOSPITAL. MEADDTONE.

ON April 3rd I was asked to see a boy, 10 years of age, who was suffering from sickness and abdominal pain. $\mathrm{He}$ had been quite well until the previous day, when he sat down with the rest of the family to breakfast. Suddenly, before taking any food, he complained of abdominal pain and vomited. The vomiting had continued at frequent intervals up to the time of my seeing him, that is, at 6.30 P.M. next day. There had been a good action of the bowels on the 1 st and no recent constipation or diarrhœa; neither flatus. mucus, nor blood had been passed since this action. The child was lying with his legs drawn up, his face was flushed, and his tongue was dry and brown. The pulse-rate was
116 and the temperature was $98^{\circ} \mathrm{F}$. He complained of 
pain in the right side of the abdomen. On examination the abdomen was seen to be flat, with the exception of a slight but distinct prominence on the right side. In the area thus defined a tumour could be felt, about two inches in length by an inch in widtb, lying with its long axis at right angles to the middle-third of a line from the umbilicus to the right anterior superior spine of the ilium. It was firm and tender and dull to percussion. Nothing else abnormal could be felt though the abdominal wall was quite yielding. A rectal examination gave no information. There had been only slight tenesmus, but an attempt to defecate just before I saw him had caused much pain and had increased the vomiting. The sudden onset of the illness and the symptoms of obstruction, with the condition of the patient, strongly pointed to an intussusception. The early and persistent vomiting and the position of the tumour suggested the ileum as the part affected. I therefore directed his friends to take him at once to the West Kent Hospital at Maidstone, his father being a labourer with a large family. At 9 P.M. I saw him in the hospital. The swelling in his abdomen previously noticed had entirely disappeared but now a somewhat indefinite mass could be feit in the position of the ascending colon. His pulse-rate was 132 and he had been again very sick. Operation was decided upon and at 9.30 r.M. I opened the abdomen by an oblique incision two and half inches in length parallel to the fibres of the external oblique muscle and one and a half inches from the

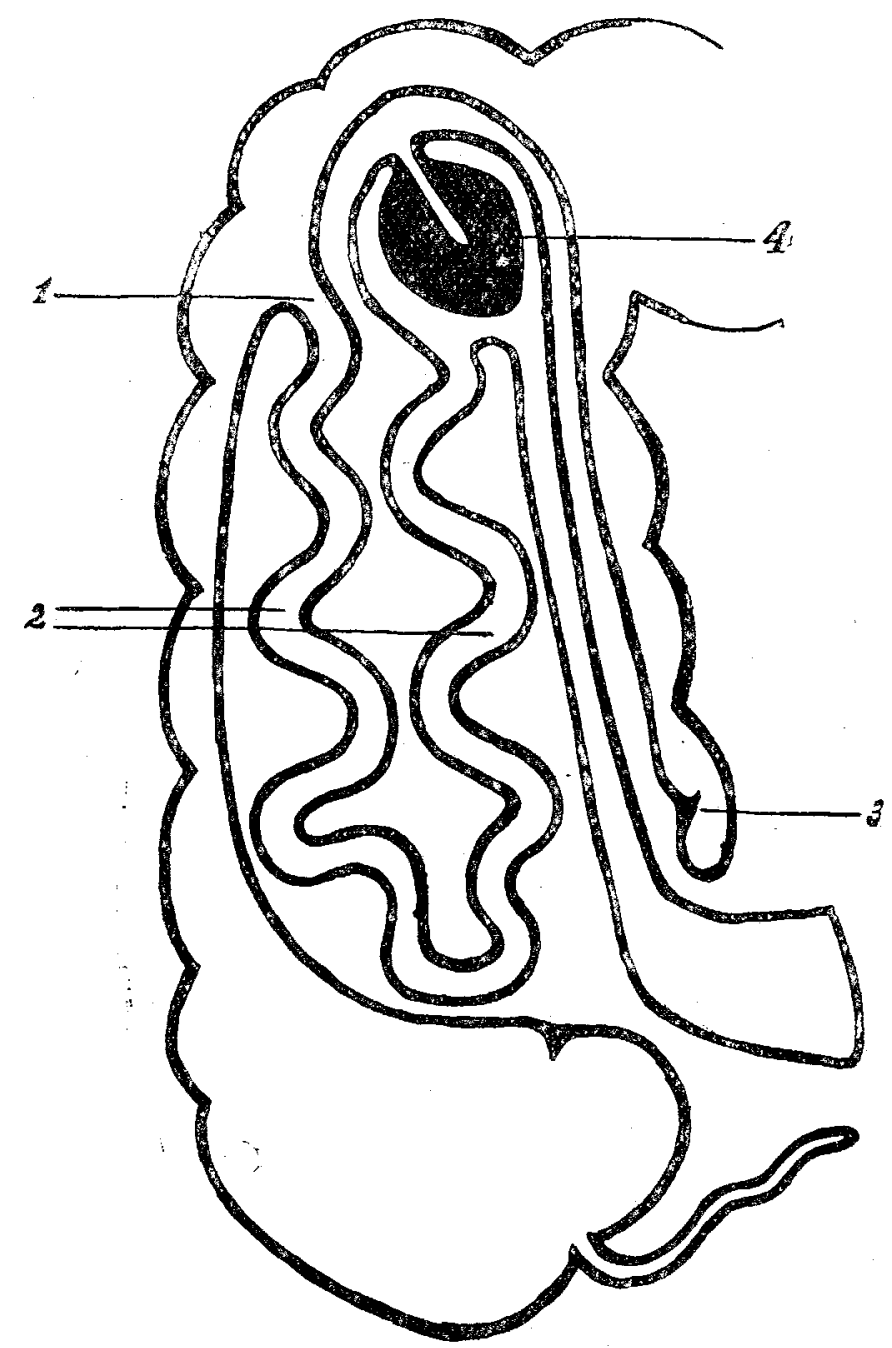

Diagram illustrating intussusception due to an inverted fiverticulum. 1, True apex of the intussusception. 2 Ileum, 14 inches, in tightly-packed coils. 3, Position of the ileo-cecal valve. 4, Inverted diverticulum, showing redematous thickening of the walls.

iliac spine. The external oblique was split and the deeper layers were drawn aside and slightly notched. On opening the peritoneum the cæcum presented; it was quite flaccid and empty. This and the long vermiform appendix were rapidly examined. The ascending colon was then felt and was found to contain a doughy mass extending for three and a half inches and with a hard lump of the size of a filbert at its apex. The ileum was hooked up with the finger and was found to be intussuscepted into the colon. By gently pressing down the mass in the colon the ileo-crecal valve was the base of the tumour. The ileum appeared to go directly to. the harder portion mentioned and by carefully pushing this down and drawing out the ileum some three inches of gut and then this mass were reduced. The passage of this portion through the ileo-creal valve was effected by pushing it through from the distal side and was the only part which gave any trouble. As soon as it was free a number of small coils of bowel easily followed which had been packed in closely and which came out much as string comes from the inside of a ball. Finally, the returning layer formed by the last three inches of the ileum was reduced. This portion of bowel showed signs of having been greatly distended and the peritoneum was sutured in two places where there were lacerations. The adjoining part of the ileum, 14 inches in length, was on reduction much compressed and anæmic but in a very few minutes it resumed a normal appearance. Above this, 18 inches from the valve, was the swollen part. On examining this it was found that a pouch or diverticulum was here intussuscepted, or rather inverted into the bowel, forming a polypoid mass which almost completely obstructed the lumen of the gut. This was partially replaced but owing to the swelling of its coats could not be entirely reduced, its apex still remaining tucked in though it no longer projected within the bowel. The diverticulum, about three-quarters of an inch in length and nearly of the size of the little finger, sprang from the gut opposite to its mesenteric attachment. A point, however, which drew my attention was the presence of a fold nearly half an inch wide running from the mesentery around the lower surface of the bowel to the little diverticulum, the edge of the fold being free and forming a little recess above and below in the angles between the ileum and the pouch. The bowel was replaced and the wound was closed. buried sutures uniting the cut muscular walls. The operation lasted just half an hour, the patient rallied well from the ordeal, and the sickness ceased entirely. The bowels acted on the 5th after a small enema. The tongue, though moist, continued to exhibit a thick fur until after the administration of several 10-grain doses of sulphate of magnesia on the 6th. The stitches were removed on the 13th and the boy was sitting up in bed on the 18th and he returned to his home on May 2 nd.

The condition here appears to have been primarily due to inversion of the diverticulum which then formed a polypoid mass and induced irregular peristalsis and secondary intussusception. The cedematous state of this pouch and the bowel wall immediately adjoining it showed that it had been affected longest; indeed, at this point alone was there any cedema present. The secondary intussusception was, I think, when first seen still of the simple enteric form and the tumour then felt was altogether on the proximal side of the ileo-cæcal valve. The change of position and shaking consequent on the removal of the patient produced the condition found at the operation, the enteric intussusception having become ileo-cæcal. The difference in the conditions of the abdomen at 6.30 P.M. and at 9 P.M. was very striking. The stretched appearance of the returning layer of the ileum and the injury to its peritoneal coat can readily be accounted for-although from the absence of cedema or circulatory disturbance the part cannot have been long involved-by the fact that into between three or three and a half inches of inverted ileum some 18 inches of the adjoining bowel and the mass formed by the inverted diverticulum had been tightly packed. The coils of bowel were wedged in by the latter which had been carried right up to the apex of the tumour.

The total absence of the classical symptom of the passage of blood and mucus is noteworthy, and the almost complete freedom from tenesmus exemplifies well how dependent this is on the implication of the large bowel in the affection, which here apparently occurred quite late.

The diagram gives almost exactly the arrangement of the bowel in the tumour, the tightly-packed coils of bowel being partly omitted for the sake of clearness.

Mairlstone.

The Water-supply of Newport (Mon.).-At the monthly meeting of the Newport corporation held on July 8th it was reported that the supply of water in the reservoir was less by $14,000,000$ gallons than for the corresponding period of last year. The service has been suspended during several hours each night for over 12 months and the shrinkage now going on foreshadows a very severe situation at the end of the summer. 